\title{
ARTICLE OPEN Flexible distributed feedback lasers based on nanoimprinted cellulose diacetate with efficient multiple wavelength lasing
}

José R. Castro Smirnov ${ }^{1}$, Ahmad Sousaraei ${ }^{1}$, Manuel R. Osorio ${ }^{1}$, Santiago Casado ${ }^{1}$, Jaime J. Hernández (iD) ${ }^{1}$ Longfei Wu ${ }^{1}$, Qi Zhang ${ }^{1,2}$, Ruidong Xia ${ }^{2}$, Daniel Granados ${ }^{1}$, Reinhold Wannemacher ${ }^{1}{ }^{1}$, Isabel Rodriguez ${ }^{1}$ and Juan Cabanillas-Gonzalez ${ }^{1}$

Here we present the assembly of novel transparent all-polymer distributed feedback (DFB) lasers. Flexible and highly transparent cellulose diacetate $(\mathrm{CdA})$ was employed as substrate on which gratings with different periods were engraved by thermal nanoimprinting with high fidelity. Highly luminescent conjugated polymers (CP), poly (9,9-dioctylfluorene) (PFO), poly(9,9dioctylfluorene-alt-benzothiadiazole) (F8BT), and a blend of F8BT and poly(3-hexylthiophene)-poly(9,9-dioctylfluorene-altbenzothiadiazole) (P3HT:F8BT) were deposited by spin coating onto the nanostructured plastic surfaces, giving rise to perpendicular single-mode lasing emission in the blue, green, and red wavelength ranges, respectively. These lasers show linewidths below $1 \mathrm{~nm}$ and low thresholds $\left(\approx 6 \mu \mathrm{Jm}^{-2}\right.$ for blue and red lasing emission), comparable to other state-of-the-art lasers obtained from similar optical gain materials on rigid substrates. The followed strategy is scalable and versatile, enabling the development of large area nanoimprinted DFB lasers $\left(>1 \mathrm{~cm}^{2}\right)$ on plastic, which is highly relevant for applications in various markets.

npj Flexible Electronics (2019)3:17; https://doi.org/10.1038/s41528-019-0062-4

\section{INTRODUCTION}

Interest in conjugated polymers (CPs) increased significantly in the last decades owing to their exceptional optical and semiconducting properties, giving rise to the emergent fields of plastic electronics ${ }^{1}$ and photonics. ${ }^{2}$ These materials combine the benefits of cost-effective processability, compatible with large-area deposition, with the distinctive mechanical features of plastics. The latter opens up ways for designing/fabricating laser geometries of virtually any shape, not possible with rigid inorganic semiconductor materials. In pursuit of this perspective, nanoimprint lithography $(\mathrm{NIL})^{3,4}$ has been used in the past to replicate different periodical surface corrugations, on thermoplastic substrates ${ }^{5}$ or on the active conjugated layers themselves. ${ }^{6}$ Standing out from other patterning techniques, NIL faithfully reproduces geometric surface patterns with sub- $5 \mathrm{~nm}$ feature size resolution ${ }^{7}$ and with high throughput that can be scaled up via roll-to-roll ${ }^{8,9}$ for low-cost mass production in a large variety of applications. ${ }^{10}$

The photoluminescence spectra of CPs can be chemically tuned, enabling optically pumped CP lasers with emission across the visible and near infrared spectrum. ${ }^{11-13}$ These highly luminescent materials have been incorporated into a variety of resonator geometries like microrings, ${ }^{14}$ photonic crystals, ${ }^{15}$ distributed Bragg reflectors (DBR), ${ }^{16}$ and distributed feedback (DFB) lasers. ${ }^{17}$ DFB lasers make use of periodic wavelength-scale nanostructures to backscatter photons within the plane of propagation. Constructive interference of the backscattered photons occurs when the grating periodicity $(\Lambda)$ fulfills the Bragg law:

$m \lambda=2 n_{\text {eff }} \Lambda$,

where $m$ is the diffraction order, $n_{\text {eff }}$ is the effective refractive index of the waveguiding medium, and $\lambda$ is the emission wavelength. All DFB lasers presented in this work are optimized to operate with the second order of diffraction $(m=2)$, with the first order of diffraction providing outcoupling of laser emission perpendicular to the plane of propagation. A wide range of applications are known for DFBs, for example in lab-on-a-chip devices for biomedical analysis, ${ }^{18}$ information technology, ${ }^{19}$ and sensing. ${ }^{20}$ Owing to their highly luminescent and flexible properties, CPs have been applied in the past for the development of mechanically flexible DFB lasers obtained with different fabrication methods. ${ }^{21-26}$ In this work we report on DFBs based on a very simple low-cost and easily scalable method resulting in samples of high optical quality and large transparency. DFB structures were fabricated by nanoimprinting gratings of short (SP, $\Lambda=287 \mathrm{~nm})$, medium, (MP, $\Lambda=350 \mathrm{~nm})$, and long period ( $\mathrm{LP}, \Lambda=416 \mathrm{~nm}$ ) on cellulose diacetate $(\mathrm{CdA})$ films and subsequent coating with PFO, F8BT, and a blend of F8BT and P3HT, respectively. For this purpose, silicon master molds were developed by electron beam lithography and subsequent reactive ion etching. The imprinted $\mathrm{CdA}$ substrates faithfully reproduce the ones of the master molds, demonstrating the great accuracy of the fabrication route followed. To the best of our knowledge, CdA has not been reported as grating substrate in DFB structures, although it has been used in DBRs ${ }^{27,28}$ and as a host for laser dyes. ${ }^{29}$ It exhibits several advantageous features: it is cheap, readily available, flexible, and transparent even upon annealing, since it does not crystallize like, for instance, polypropylene. Moreover, CdA presents other advantages, like being compatible with several organic solvents, it is obtained from renewable wood pulp sources and it is biodegradable. The relatively low refractive index of $\mathrm{CdA}$ $(n \approx 1.475)$ leads to a $0.2-0.3$ index mismatch with most CPs, sufficient to provide substantial vertical light confinement.

\footnotetext{
${ }^{1}$ Madrid Institute for Advanced Studies in Nanoscience, IMDEA Nanociencia, Calle Faraday 9, Ciudad Universitaria de Cantoblanco, 28049 Madrid, Spain and ${ }^{2}$ Key Laboratory for Organic Electronics and Information Displays and Institute of Advanced Materials, National Jiangsu Synergistic Innovation Center for Advanced Materials (SICAM), Nanjing University of Posts and Telecommunications, 9 Wenyuan Road, Nanjing 210046, PR China

Correspondence: José R. Castro Smirnov (jose.castro@imdea.org) or Juan Cabanillas-Gonzalez (juan.cabanillas@imdea.org)
}

Received: 18 February 2019 Accepted: 29 July 2019

Published online: 03 September 2019 
a)

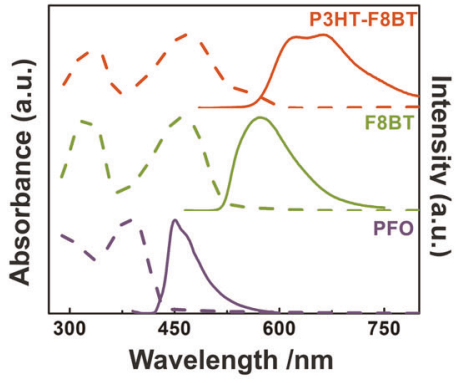

d) b)

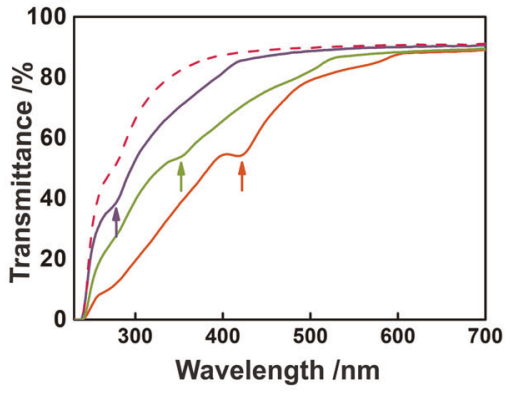

c)

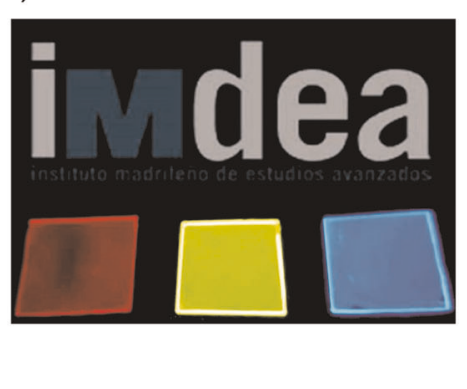

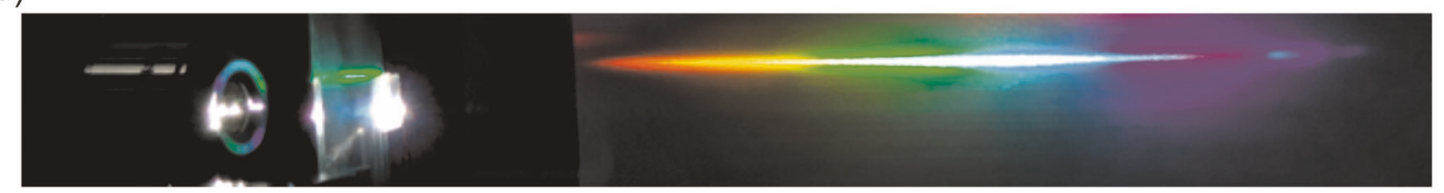

Fig. 1 a Normalized absorption and photoluminescence spectra of PFO (blue solid line and dot line), F8BT (green solid line and dot line), and P3HT:F8BT (red solid line and dot line). b Transmission spectra at normal incidence of LP (red solid line), MP (green solid line), and SP (blue solid line) gratings imprinted on $\mathrm{CdA}$ foils and of the unpatterned $\mathrm{CdA}$ substrate (pink dashed line). The arrows point at the transmittance dips, corresponding to the wavelengths of 278,350 , and $416 \mathrm{~nm}$, respectively. c PFO (right), F8BT (middle), and P3HT:F8BT (left) DFB structures illuminated by a UV lamp. d Diffraction pattern of a naked CdA grating when exposed to white light

\section{RESULTS AND DISCUSSION}

In this work we have chosen PFO, F8BT, and a blend of P3HT and F8BT, which are well known CP systems exhibiting optical gain in the blue, ${ }^{30}$ green-yellow ${ }^{31,32}$ and red $^{33}$ spectral regions, respectively. Superimposed photoluminescence and absorption spectra of the three CP media are illustrated in Fig. 1a. Unpatterned CdA exhibits large transmission $(>80 \%)$ in the visible. The transmission drop below $400 \mathrm{~nm}$ and the dip at $280 \mathrm{~nm}$ (dashed line in Fig. 1b) is assigned to the $n \pi^{*}$ transition of the acetate keto group as previously described. ${ }^{34}$

The transmission spectra of SP, MP, and LP patterned CdA display additional losses at wavelengths located below 420, 531, and $605 \mathrm{~nm}$, respectively, caused by diffraction. These limit wavelengths stand for the grating cut-off values, i.e., the appearance of first order diffraction under $90^{\circ}$ on the $\mathrm{CdA}$ side of the nanoimprinted grating $\left(\frac{\lambda}{n} \approx \Lambda\right)$. Likewise, dips are observed at wavelengths marked by arrows, $(278,350$, and $417 \mathrm{~nm}$, respectively), likely due to first order diffraction under $90^{\circ}$ at the air side of the grating $(\lambda \approx \Lambda)$. CP films were spin coated on top of these nanoimprinted $\mathrm{CdA}$ substrates. In Fig. 1c, the homogeneity and luminescent properties of these films under UV illumination is illustrated. Such good homogeneity is a consequence of the high surface energy of the CdA substrates, ${ }^{35}$ which are readily wetted by the $C P$ solution in toluene. Figure $1 d$ depicts a diffraction pattern obtained upon illuminating an uncoated $\mathrm{CdA}$ grating with white light.

The fidelity of the pattern transfer after the imprint process was assessed by comparing the atomic force microscopy (AFM) topography images of corrugated silicon and $\mathrm{CdA}$ substrates (Fig. 2). The grating depth profile for each mold is also shown. The fact that there are no major differences between the silicon master mold and the CdA grating indicates the high fidelity of the soft patterning. A cross-sectional profile across ten grooves in each surface provides local periods of 275 (283), 351 (354), and 403 (403) nm for the SP, MP, and LP Si (CdA) corrugated surfaces, respectively, confirming less than $3 \%$ of variation in periodicity in all cases. The surface profiles extracted from each AFM image indicate similar modulation depths of the grating structures in $\mathrm{Si}$ and CdA of about $120 \mathrm{~nm}$ for the SP, $140 \mathrm{~nm}$ for the MP, and $250 \mathrm{~nm}$ for the LP gratings, respectively.
Figure 3 illustrates front views of CdA SP, MP, and LP gratings (Fig. 3a-C, respectively) and a side view of a CdA SP grating (Fig. 3d) obtained by scanning electron microscopy (SEM).

Note that the height of the modulation in Fig. $3 d(247 \mathrm{~nm})$ is compatible with the height profile measured by AFM. The quality of the nanoimprinted gratings on $\mathrm{CdA}$ was further evaluated employing grazing incidence small angle $\mathrm{X}$-ray scattering (GISAXS), a technique that has been employed previously for the characterization of gratings prepared by different methods. ${ }^{36,37}$ Unlike AFM, the 2D GISAXS patterns provide statistical information averaged over a larger surface area covered by the footprint of the X-ray beam. In our experiments, the very low incident angle $\left(0.3-0.4^{\circ}\right)$ and the cross-section area of the X-ray beam $(1.3 \mathrm{~mm} \times 0.3 \mathrm{~mm})$ implied a probing area of $\sim 70 \mathrm{~mm}^{2}$. The top row of the figure shows the corresponding X-ray diagrams obtained from the Si gratings. The patterns show an array of rods that correspond to different orders of scattering located at horizontal scattering angles $\omega$, which are reciprocally related to the pitch of the periodic nanostructures. These rods intersect the Ewald sphere on a series of dots distributed along a semicircle. Such GISAXS patterns are characteristic for scattering from gratings at very low incidence angles. ${ }^{38,39}$ The semicircular pattern characteristic of Si gratings vanishes in the CdA replica, most likely due to its reduced planarity, being instead composed of vertical stripes. Figure $4 \mathrm{~b}$ shows the 1D profiles obtained after integration of the vertical scattered intensities along the whole $\omega$ range. For the sake of clarity, only one half of the curve is presented. The curves were shifted to correct the pixel position according to the origin $(\omega=0)$, estimated by the symmetry of the reflections on both sides of the beam stop. Despite of being qualitatively different, the profile obtained from the $\mathrm{CdA}$ grating patterns reproduce rather well the profile of the corresponding $\mathrm{Si}$ molds, confirming that the periodicity of the structure is faithfully transferred to the polymer substrate over a large area during the imprinting process.

The periods of each grating were calculated as the mean of those obtained from different reflection orders, according to the equations described in the methods section. Table 1 provides a comparison of the periods obtained for the Si molds and the CdA replica, respectively. The obtained values are in good agreement with those observed by AFM and SEM. 
a)
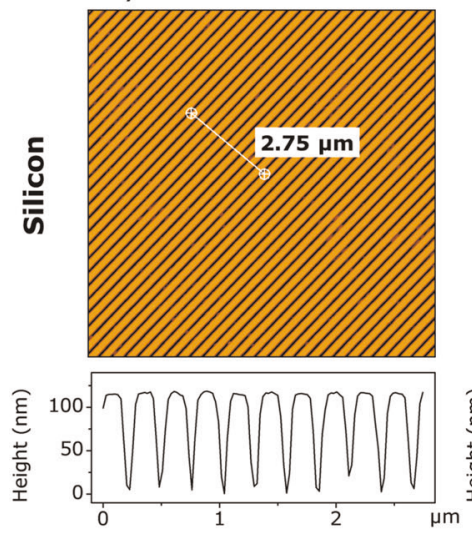

b)
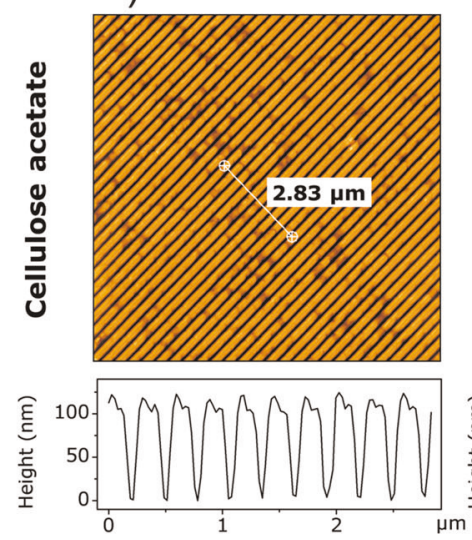

c)

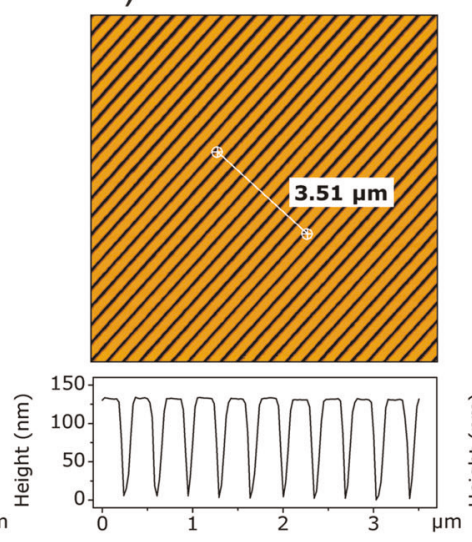

d)
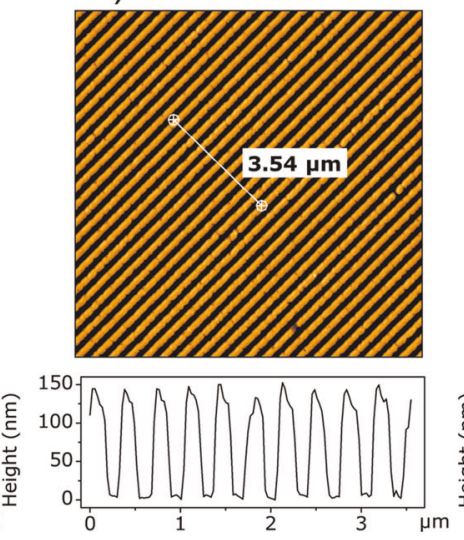

e)

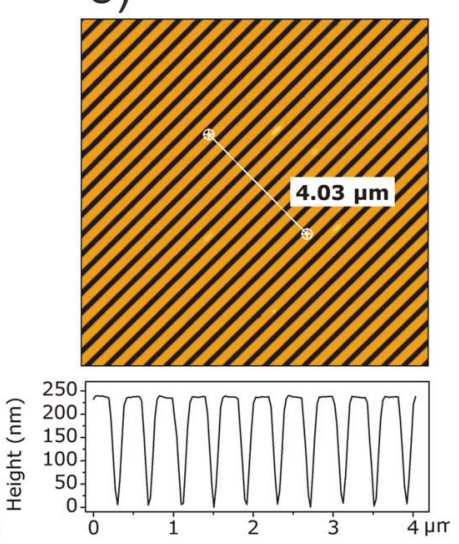

f)
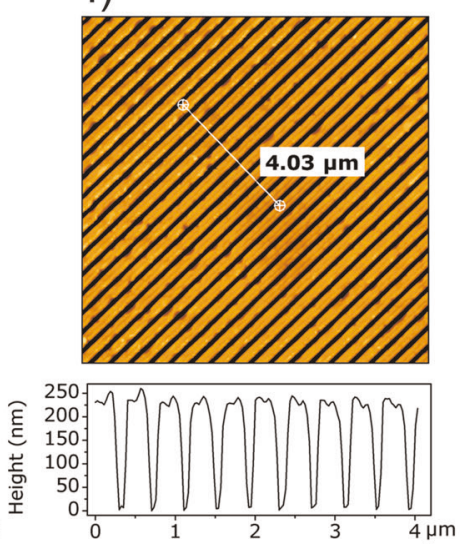

$200 \mathrm{~nm}$

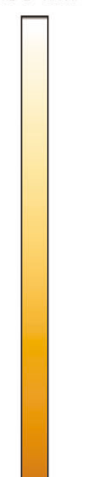

$0 \mathrm{~nm}$

Fig. 2 AFM topography images and profiles along the lines indicated in the images of grating structures fabricated in Si and in CdA films, with $\mathrm{SP}(\mathbf{a}, \mathbf{b}), \mathrm{MP}(\mathbf{c}, \mathbf{d})$, and LP (e, f)

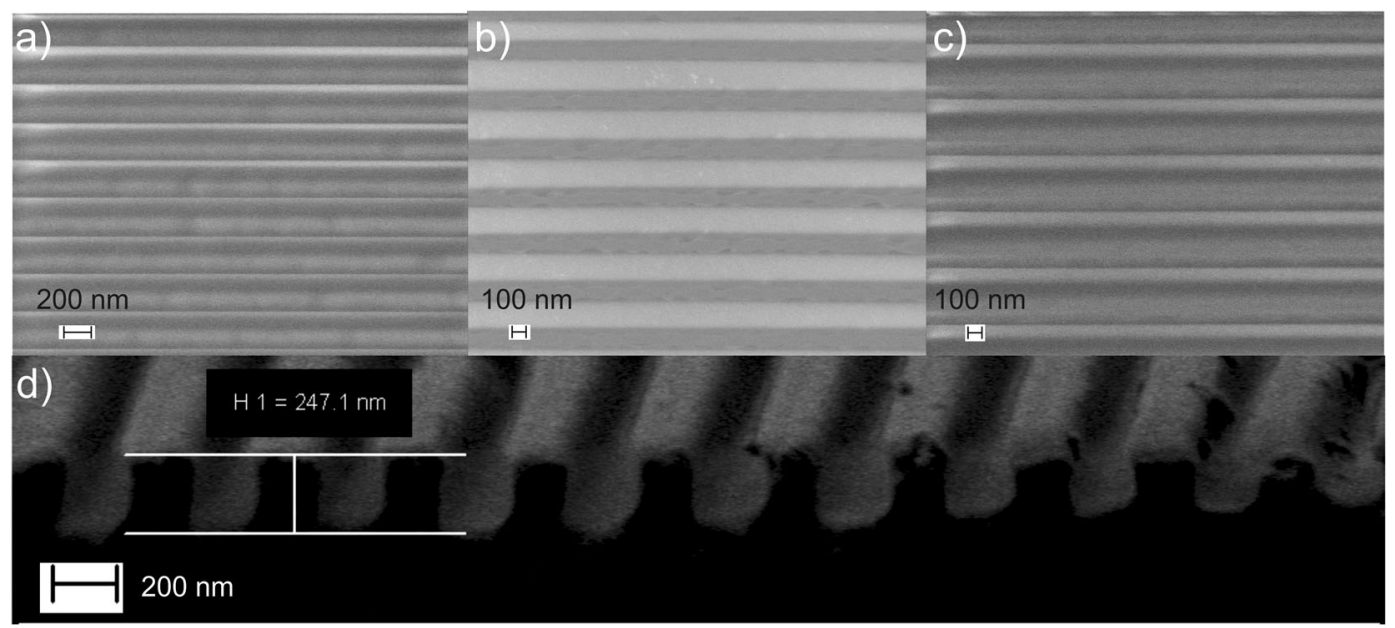

Fig. 3 SEM images of gratings structures fabricated in CdA films, with a SP, b MP, and $\mathbf{c}$ LP. $\mathbf{d}$ Cross section of an LP CdA grating

Figure 5 shows the emission spectra of (a) PFO, (b) F8BT, and (c) P3HT:F8BT films deposited on top of corrugated CdA substrates at excitation densities below and above the lasing thresholds, as well as the corresponding input-output curves of emission intensity vs. pump fluence.

The dotted lines represent the normalized emission spectra at pump fluence just below the respective laser thresholds. The blue, green, and red solid lines, on the other hand, represent lasing spectra for pump fluences just above the thresholds, detected in the direction perpendicular to the surface. These spectra are characterized by narrow peaks at 450, 576, and $676 \mathrm{~nm}$, respectively, with linewidth values of $0.2,0.4$, and $0.3 \mathrm{~nm}$. In all cases, the laser signals are emerging from dips surrounded by two fluorescence peaks. The dip is explained by the presence of a photonic stopband, caused by the distributed feedback (DFB) of the propagating modes in the corrugated films. The lasing 
a)

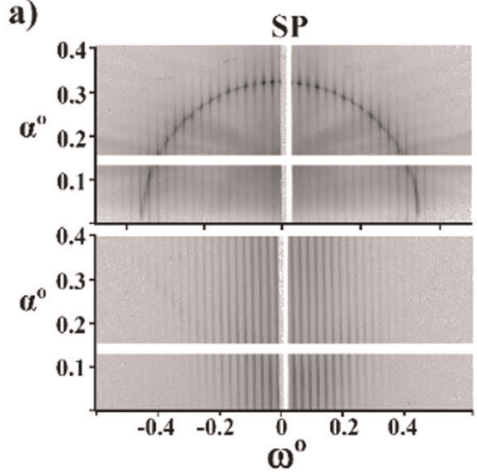

b)

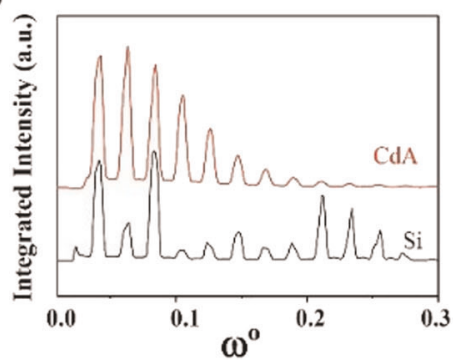

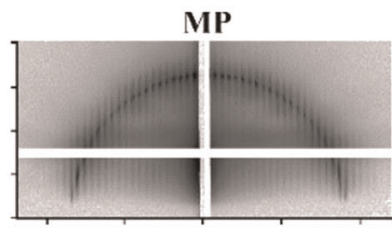
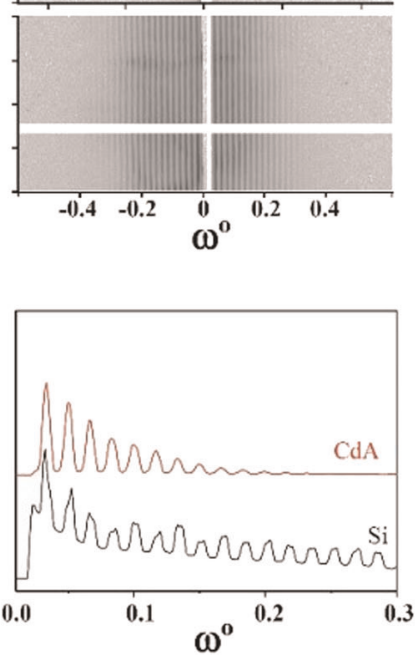
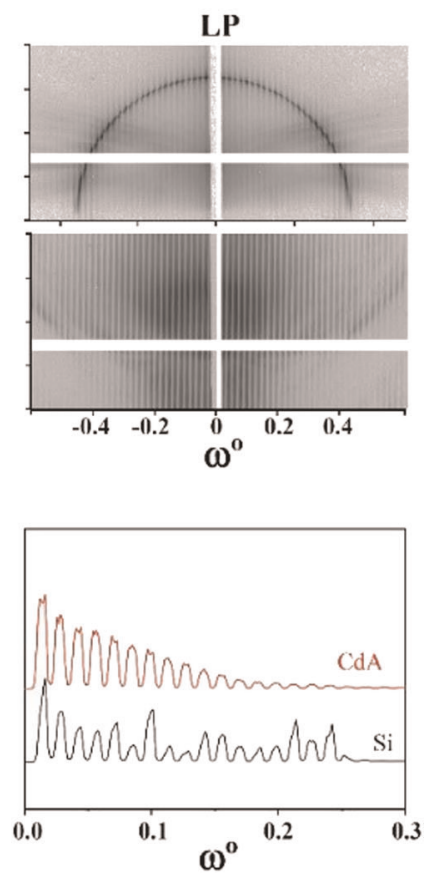

Fig. 4 a 2D GISAXS patterns corresponding to the Si gratings (top row) and to the replica prepared by NIL in CdA foil (bottom row). $\mathbf{b} 1 \mathrm{D}$ integrated X-ray intensity for the Si molds (black) and CdA replica (red) obtained by integrating the intensity along the vertical direction of the GISAXS. In order to facilitate the comparison between the periods of the Si mold and the CdA copy only one half of the curve is presented

Table 1. Grating periods determined from GISAXS diffraction patterns of $\mathrm{Si}$ molds and the corresponding CdA replica

\begin{tabular}{ll}
\hline Sample & Period $(\mathrm{nm})$ \\
\hline SP Si & $276 \pm 2$ \\
MP Si & $352 \pm 3$ \\
LP Si & $417 \pm 5$ \\
SP CdA & $276 \pm 5$ \\
MP CdA & $355 \pm 7$ \\
LP CdA & $409 \pm 10$ \\
\hline
\end{tabular}

thresholds of the plastic DFB lasers, $\left(6.4 \mu \mathrm{Jcm}^{-2}\right.$ for blue, $54.0 \mu \mathrm{Jcm}^{-2}$ for green-yellow, and $6.6 \mu \mathrm{Jcm}^{-2}$ for red emission, respectively), are extracted from the drastic variation in the slopes of the output emission intensities (see Fig. $5 d-f$ ). The thresholds obtained are comparable to those previously reported on similar blue, ${ }^{17,40}$ green-yellow, ${ }^{41,42}$ and red $^{43,44}$ DFBs on nanostructured rigid substrates as well as on nanostructured photoresists. ${ }^{45,46}$ The slopes of the input-output curves for F8BT and F8BT:P3HT are 5.5 and 50 times lower, respectively, than the corresponding slope for PFO, which suggests superior optical gain properties in PFO. This is consistent with the larger gain coefficient obtained from ASE measurements in PFO $\left(74 \mathrm{~cm}^{-1}\right)$ with respect to F8BT $\left(22 \mathrm{~cm}^{-1}\right)$ using the variable slit method. ${ }^{47}$ Indeed, tuning of the emission of polyfluorenes to longer wavelengths via backbone functionalization results in a progressive increase of spectral overlap between stimulated emission and excited-state absorption absorption, which is detrimental for optical gain. ${ }^{48}$ Regarding F8BT:P3HT blends the lower slope efficiency could be explained as due to the lesser emissive properties of the blend respect to PFO and F8BT $(24,50$, and $58 \%$ photoluminescence quantum efficiency values of F8BT:P3HT, PFO, and F8BT, respectively). ${ }^{47,49}$ Tunability of the emission from the DFB structures was achieved through control of the active layer thickness by varying the spin coating speed or the $\mathrm{CP}$ concentration in precursor solutions. The shift in DFB emission with thickness can be explained as due to the change in the effective refractive index of the planar waveguide, which increases toward the refractive index of the active medium when the film thickness increases. Changing the PFO and P3HT:F8BT film thicknesses from 142 to $232 \mathrm{~nm}$ and from 120 to $260 \mathrm{~nm}$ led consequently to a displacement of the emission peaks to longer wavelengths by up to 12 and $34 \mathrm{~nm}$, respectively (Fig. 6).

Finally, we investigated the laser emission properties upon bending the substrate at three different angles: $41^{\circ}, 61^{\circ}$, and $113^{\circ}$ (Fig. 7). Increasing the bending angle leads to a progressive emission blue shift, (Fig. 7a). We tentatively assign this effect to an effective decrease in thickness of the waveguiding medium leading to the aforementioned shift of the lasing mode. Importantly, the lasing threshold values of bent DFBs did not differ substantially from that of an undeformed DFB (Fig. 7b), confirming that the optical and structural properties of the active layer do not deteriorate.

In summary, we report a straightforward fabrication method of flexible DFB lasers that involves NIL on inexpensive transparent $\mathrm{CdA}$ substrates. By spin coating different CPs on periodically patterned $\mathrm{CdA}$ we have achieved optically induced vertical singlemode laser emission in the blue, green-yellow, and red wavelength range. The described procedure enables the mass fabrication of low-cost and large area diffractive elements on plastic for lighting, sensing, and lab-on-a-chip applications. The fact that these DFBs are deployed on thin and transparent substrates provides the option to pump them simultaneously by stacking them on top of each other, giving rise in this way to multicolored laser emission.

\section{METHODS}

Fabrication of the $350 \mathrm{~nm}$ Si mold

Silicon master molds with periods of 278 and $416 \mathrm{~nm}$ were acquired from Lightsmyth Technologies Inc. The silicon master mold with a period of $350 \mathrm{~nm}$ was fabricated by electron beam lithography and reactive ion etching techniques. First of all the pattern was transferred to a PMMA layer of about $180 \mathrm{~nm}$ in thickness, directly spun onto the silicon substrate. The 
(a)

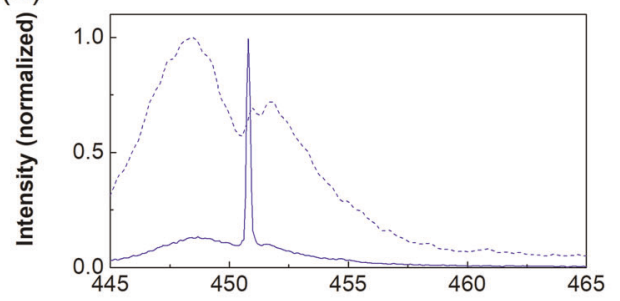

(b)

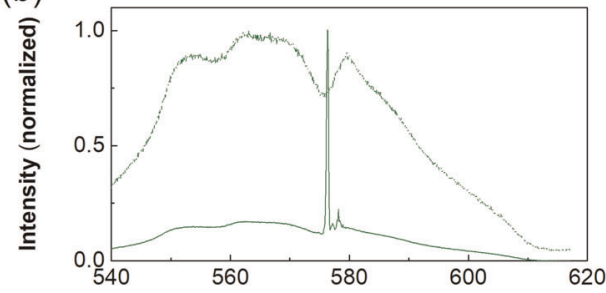

(c)

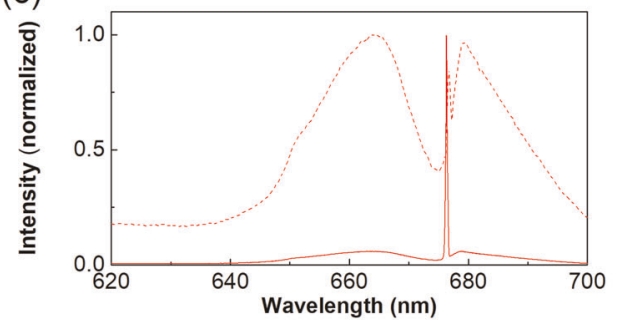

(d)

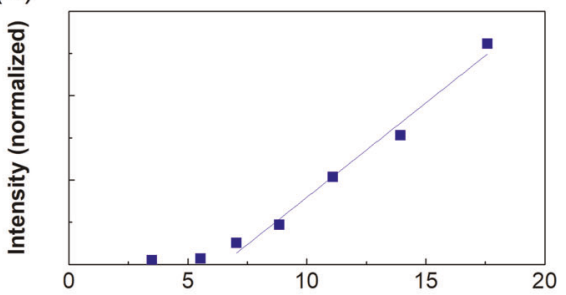

(e)

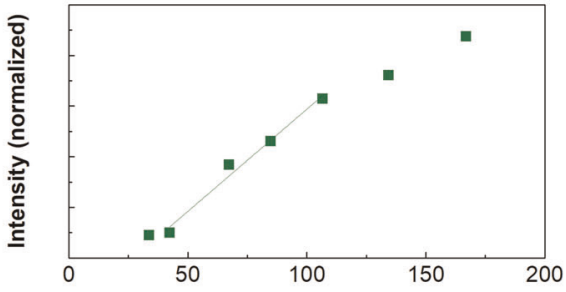

(f)

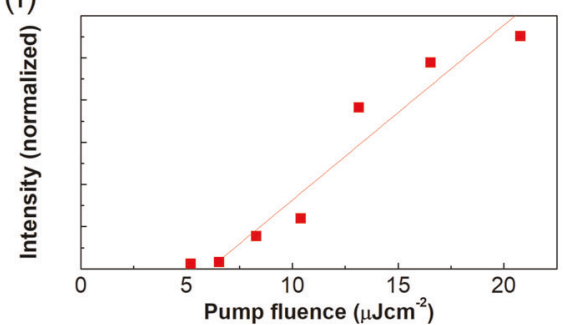

Fig. 5 Left column: Normalized intensity of a blue, $\mathbf{b}$ green-yellow, $\mathbf{c}$ red, emission from the CP DFB lasers below the threshold (dashed lines) and just above the threshold (solid lines). Right column: Variation of the output laser power as a function of pump fluence in $\mathbf{d}$ blue, $\mathbf{e}$ green-yellow, and f red emitting DFB structures. The red, green, and blue solid lines represent linear fits above the thresholds

(a)

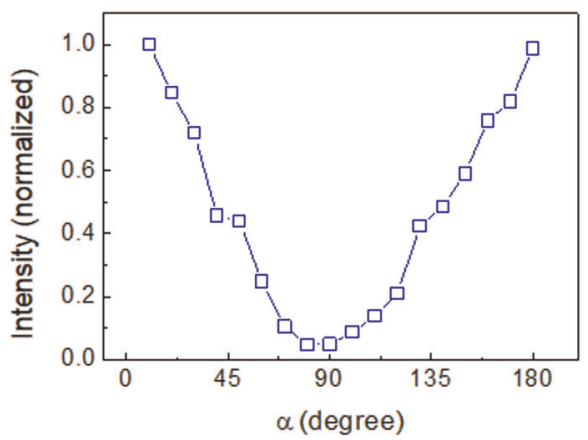

(b)

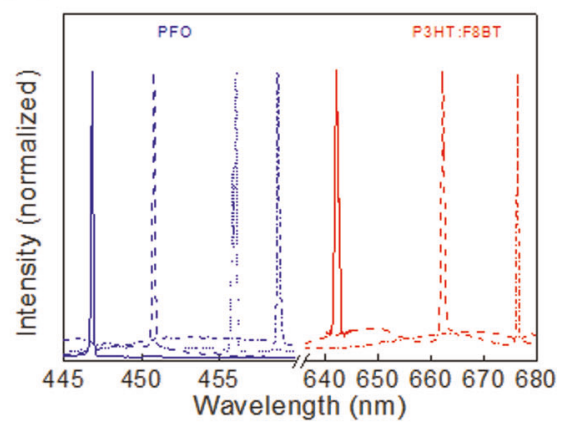

Fig. 6 a Polarized emission output of a DFB based on F8BT. The pump polarization was fixed along the DFB lines, whereas the output intensity was filtered with a polarizer at different angles $(a)$. b Normalized output spectra of PFO and P3HT:F8BT DFB structures above threshold. PFO concentrations: $10 \mathrm{mgml}^{-1}$ (blue solid line line), $20 \mathrm{mgml}^{-1}$ (blue dash line), $30 \mathrm{mgml}^{-1}$ (blue short dot line), and $40 \mathrm{mgml}^{-1}$ (short dash dot line). P3HT:F8BT concentrations: $10 \mathrm{mgml}^{-1}$ (red solid line line), $20 \mathrm{mgml}^{-1}$ (red dash line), and $40 \mathrm{mgml}^{-1}$ (red short dash dot line). The corresponding PFO film thicknesses were 142, 178, 206, and $232 \mathrm{~nm}$, as measured by AFM. The corresponding P3HT:F8BT film thicknesses were 120,180 , and $260 \mathrm{~nm}$

resist was exposed by using an Auriga SEM (Carl Zeiss) controlled by an ELPHY MultiBeam lithography system (Raith $\mathrm{GmbH}$ ). After developing the sample in MIBK:IPA (1:3) and rinsing it in IPA, as usual, the sample went through a soft oxygen $50 \mathrm{~W}$ plasma process during $15 \mathrm{~s}$ to remove any possible residues of resist in the developed areas. The reactive ion etching was performed with an Oxford Instruments Plasmalab System 100. As the required depth was about $150 \mathrm{~nm}$ and the selectivity of silicon to PMMA is as high as $5: 1$ at about $20^{\circ} \mathrm{C}$, the etching process was performed at ambient temperature. A short run of $17 \mathrm{~s}$, with $10 \mathrm{sccm}$ of SF6 for etching and $63 \mathrm{sccm}$ of CHF3 for sidewall passivation, $20 \mathrm{mTorr}, 20 \mathrm{~W}$ of forward power, and $220 \mathrm{~W}$ for the ICP were enough to obtain the desired depth.
Nanoimprint of gratings on $\mathrm{CdA}$ substrates

The Si DFB master molds were initially treated with a fluorosilane through vapor deposition of $1 \mathrm{H}, 1 \mathrm{H}, 2 \mathrm{H}, 2 \mathrm{H}$-perfluorodecyl trichlorosilane (Alfa Aesar, $M A)$ as release agent to reduce the surface energy, easing the demolding procedure. Next, the master mold was imprinted directly onto a CdA film by means of a Eitre 3 Nano Imprint Lithography System by Obducat. As the $\mathrm{CdA}$ film is flexible, it perfectly adjusts to surface irregularities, therefore leading to very homogeneous imprint nanopatterns. Throughout the nanoimprinting route, substrates were heated at $180^{\circ} \mathrm{C}$ with an applied pressure of $40 \mathrm{Bar}$ for $300 \mathrm{~s}$. At the imprinting temperature the polymer 
(a)

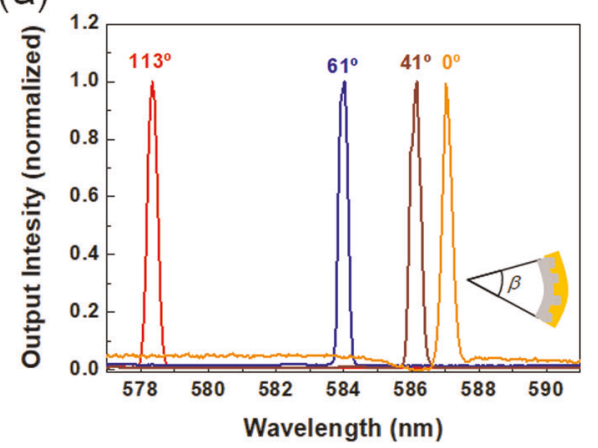

(b)

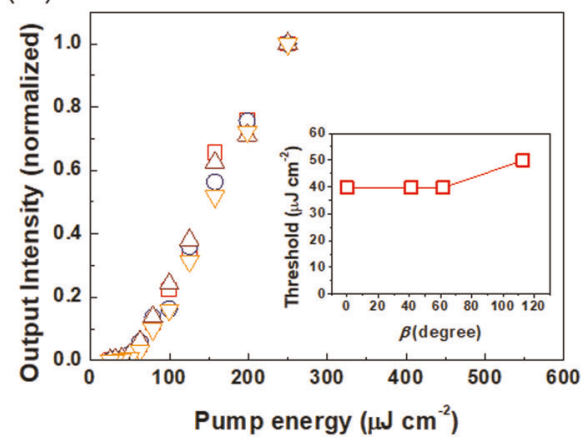

Fig. 7 a Laser emission on DFBs based on F8BT upon $113^{\circ}$ bending (red), $61^{\circ}$ (blue), $41^{\circ}$ (brown), and $0^{\circ}$ (orange). The inset sketch illustrates a DFB under a $\beta$ bending angle. $\mathbf{b}$ Integrated emission intensity as function of pump fluence for different bending angles: $113^{\circ}$ (squares), $61^{\circ}$ (circles), $41^{\circ}$ (up-triangles), and $0^{\circ}$ (down-triangles). Lasing thresholds as a function of bending angle are shown in the inset

softens and fills the mold cavities. The substrate was then cooled down to a temperature of $70^{\circ} \mathrm{C}$ before the pressure was released and the imprinted substrate was removed. Three grating structure varieties were used: (i) $\Lambda=$ $416 \mathrm{~nm}$ period, fill-factor $50 \%$, and $250 \mathrm{~nm}$ etch depth, (ii) $\Lambda=350 \mathrm{~nm}$, fillfactor $50 \%$, and $150 \mathrm{~nm}$ etch depth, and (iii) $\Lambda=278 \mathrm{~nm}$, fill-factor $50 \%$, and $110 \mathrm{~nm}$ etch depth.

\section{Fabrication of all-polymer DFB}

As starting materials, a flexible CdA film (refractive index: $n=1.470$ at $676 \mathrm{~nm}, n=1.475$ at $576 \mathrm{~nm}$, and $n=1.482$ at $450 \mathrm{~nm})^{50}$ of $300 \mu \mathrm{m}$ thickness with flat surfaces provided by Clarifoil (Celanese, UK) was selected as a substrate. Highly luminescent conjugated polymers poly $(9,9-$ dioctylfluorene-alt-benzothiadiazole) (F8BT) (American Dye Source, refractive index ${ }^{51} n=1.76$ at $\left.576 \mathrm{~nm}, M n=33 \mathrm{kgmol}^{-1}, M w=217 \mathrm{kgmol}^{-1}\right)$; poly (9,9-dioctylfluorene) (PFO, American Dye Source, refractive index $n=$ 1.81 at $\left.450 \mathrm{~nm}^{51} \mathrm{Mn}=40-150 \mathrm{kgmol}^{-1}\right)$, and a blend of poly(3hexylthiophene) (P3HT) (BASF Sepiolid P200, $>98 \%$ regioregularity, $M n=$ $\left.10 \mathrm{kgmol}^{-1}\right)$ and poly(9,9-dioctylfluorene-alt-benzothiadiazole) (P3HT: F8BT) (refractive index ${ }^{43}: n=1.80$ at $676 \mathrm{~nm}$ ), were dissolved in toluene at a concentration of $20 \mathrm{mgml}^{-1}$ and the resulting solutions were filtered with a $0.22 \mu \mathrm{m}$ syringe filter. P3HT:F8BT solution mixtures were prepared upon mixing master toluene solutions of $20 \mathrm{mgml}^{-1}$ regioregular P3HT with F8BT in a weight concentration of $20 \mathrm{mgml}^{-1}$. All CP films were spin coated at $2000 \mathrm{rpm}$ for $1 \mathrm{~min}$ onto periodically corrugated CdA substrates, resulting in average thicknesses of $180 \mathrm{~nm}$ for all cases.

\section{Optical and structural characterization}

A narrow-band OPO (Continuum Sunlite, bandwidth $0.075 \mathrm{~cm}^{-1}$, pulse width $\approx 3 \mathrm{~ns}$ ) pumped by a seeded frequency-tripled $\mathrm{Nd}: \mathrm{YAG}$ laser (Continuum Powerlite PL8000) and equipped with a frequency doubler (Continuum FX-1) was used for excitation. The wavelength range accessible with this OPO is $220-1700 \mathrm{~nm}$. The pump beam was strongly attenuated for the experiments. The lasing emission arising perpendicularly from the surface of the DFBs was detected with a spectrometer (SP2500, Acton Research) equipped with a liquid nitrogen cooled backilluminated deep depletion CCD (Spec-10:400BR, Princeton Instruments). For lasing measurements, the oblique incidence pump beam was passed through a calibrated diaphragm and focused using a $30 \mathrm{~mm}$ focal length spherical lens, with the sample placed out of focus. The emission was collected in free-space normal to the DFB surface. The pumping intensity was regulated with neutral density filters. UV-Vis characterization was performed using a Varian Cary 50 UV-Vis spectrophotometer. AFM images were obtained using a JPK NanoWizard II operating in dynamic mode and air conditions, with MikroMasch $\mathrm{HQ}: \mathrm{XSC} 11 / \mathrm{Al}$ BS cantilevers of $2.7 \mathrm{~nm}^{-1}$ force constant and $80 \mathrm{kHz}$ resonance frequency. SEM characterization was carried out by means of a Carl Zeiss Auriga microscope operating at $3 \mathrm{kV}$.

\section{GISAXS measurements}

GISAXS measurements were performed at the BM26 DUBBLE Dutch-Belgian beamline of the European Synchrotron Radiation Facility. ${ }^{52}$ A sample to detector distance of $7322 \mathrm{~mm}$ was estimated using a rat tail sample as standard of calibration. The energy of the X-ray beam was fixed at $12 \mathrm{KeV}(1.03 \AA$ ) . The 2D scattering patterns were recorded by using a Pilatus $1 \mathrm{M}$ detector with $172 \mu \mathrm{m}$ pixel size. The slits alignment allowed for a beam size (horizontal $\times$ vertical) of $1.3 \times 0.3 \mathrm{~mm}$. A nominal incidence angle of $a i=0.35^{\circ}$ was selected, allowing to average the recorded information over an area of ca. $70 \mathrm{~mm}^{2}$. In order to verify the periodicity of the gratings and the quality of the polymer replica, the samples were carefully aligned, with the lines parallel to the X-ray beam, since the appearance of the diffraction pattern is very sensitive to any change in this factor. GISAXS images were analyzed by using the FIT2D software. Basically, in a GISAXS pattern there exist two main scattering angles used to obtain information about structural correlations in the vertical and horizontal directions, $a$ and $\omega$, respectively. The information can be interpreted on the basis of three orthogonal scattering vectors, $q x, q y$, and $q z$, being $q y=\left(2 \pi \lambda^{-1}\right) \sin \omega \cdot \cos a$, the one which probes the correlations existing in the horizontal plane, ${ }^{53}$ that is, the grating period under study. Once the scattering vector qy has been calculated, the period, $\Lambda$, of the grating can be extracted from the relation $\Lambda=2 \pi(q y)^{-1}$.

\section{DATA AVAILABILITY}

The datasets generated during and/or analyzed during the current study are available from the corresponding author on reasonable request.

\section{ACKNOWLEDGEMENTS}

J.C-G. is grateful to the Spanish Ministerio de Economía, Industria y Competitividad through MAT2014-57652-C2-1-R (LAPSEN), RTI2018-097508-B-I00 (AMAPOLA), and PCIN-2015-169-C02-01 (MOFSENS) projects. R.W. gratefully acknowledges support by the European Union structural funds and the Comunidad de Madrid NMAT2D-CM project (S2018/NMT-4511), as well as by the Spanish Ministerio de Economía, Industria y Competitividad through project No. MAT2015-71879-P. IMDEA Nanociencia acknowledges support from the "Severo Ochoa" Programme for Centers of Excellence in R\&D (MINECO, Grant SEV-2016-0686). R.X. thanks for financial support from the National Natural Science Foundation of China (Grants 61874058 and 61376023), the National Key Basic Research Program of China (973 Program, 2015CB932203), and the Priority Academic Program Development Fund of Jiangsu Higher Education Institutions in China. This work is partially supported by the Spanish Ministry of Economy, Industry and Competitiveness through Grant SUPERMNAN ESP2015-65597-C4-3-R DETECTA ESP2017-86582-C4-3-R. D.G. acknowledges Grant RYC-2012-09864. The authors are grateful to D. Hermida for excellent technical support during the synchrotron experiments at the BM26 beamline.

\section{AUTHOR CONTRIBUTIONS}

J.C.-G., R.W. and I.R. conceived the research, supervised the whole study, and contributed to the manuscript review. J.R.C.S. performed all experiments, measurements, and wrote the manuscript. M.R.O. fabricated the $350 \mathrm{~nm} \mathrm{Si} \mathrm{mold} \mathrm{and}$ performed SEM measurements. S.C. performed AFM measurements. A.S., Q.Z., R.X., L. W. and R.W. contributed to optical characterization and sample preparation. J.H. performed GISAXS measurements. D.G. performed the experiments to show a diffraction pattern of naked CdA gratings. All authors contributed to the discussion and analysis of the results. 


\section{ADDITIONAL INFORMATION}

Competing interests: The authors declare no competing interests.

Publisher's note: Springer Nature remains neutral with regard to jurisdictional claims in published maps and institutional affiliations.

\section{REFERENCES}

1. Forrest, S. R. The path to ubiquitous and low-cost organic electronic appliances on plastic. Nature 428, 911-918 (2004).

2. O'Carroll, D. M. et al. Conjugated polymer-based photonic nanostructures. Polym. Chem. 4, 5181-5196 (2013).

3. Chou, S. Y., Krauss, P. R. \& Renstrom, P. J. Imprint of sub- $25 \mathrm{~nm}$ vias and trenches in polymers. Appl. Phys. Lett. 67, 3114 (1995).

4. Guo, L. J. Nanoimprint lithography. methods and materials requirements. Adv. Mater. 19, 495-513 (2007).

5. Ramírez, M. G., Villalvilla, J. M., Quintana, J. A., Boj, P. G. \& Díaz-García, M. A. Distributed feedback lasers based on dichromated poly(vinyl alcohol) reusable surface-relief gratings. Opt. Mater. Express 4, 733-738 (2014).

6. Ramirez, M. G. et al. Efficient organic distributed feedback lasers with imprinted active films. Opt. Express 19, 22443-22454 (2011).

7. Schift, H. Nanoimprint lithography: an old story in modern times? A review. J. Vac. Sci. Technol. B: Microelectron. Nanometer Struct. Process., Meas., Phenom. 26, 458-480 (2008)

8. Lee, S. et al. Facile three-dimensional nanoarchitecturing of double-bent gold strips on roll-to-roll nanoimprinted transparent nanogratings for flexible and scalable plasmonic sensors. Nanoscale 9, 1398-1402 (2017).

9. Aikio, S. et al. Disposable photonic integrated circuits for evanescent wave sensors by ultra-high volume roll-to-roll method. Opt. Express 24, 2527-2541 (2016).

10. Kooy, N., Mohamed, K., Pin, L. T. \& Guan, O. S. A review of roll-to-roll nanoimprint lithography. Nanoscale Res. Lett. 9, 320 (2014).

11. Moses, D. High quantum efficiency luminescence from a conducting polymer in solution: a novel polymer laser dye. Appl. Phys. Lett. 60, 3215-3216 (1992).

12. Tessler, N., Denton, G. J. \& Friend, R. H. Lasing from conjugated-polymer microcavities. Nature 382, 695-697 (1996).

13. Samuel, I. D. W. \& Turnbull, G. A. Organic semiconductor lasers. Chem. Rev. 107, 1272-1295 (2007).

14. Kawabe, Y. et al. Whispering-gallery-mode microring laser using a conjugated polymer. Appl. Phys. Lett. 72, 141 (1998)

15. Deutsch, M., Vlasov, Y. A. \& Norris, D. J. Conjugated-polymer photonic crystals. Adv. Mater. 12, 1176-1180 (2000).

16. Peter, K. H., Ho, D., Stephen, T., Friend, R. H. \& Tessler, N. All-polymer optoelectronic devices. Science 285, 233-236 (1999).

17. Heliotis, G. et al. Emission characteristics and performance comparison of polyfluorene lasers with one- and two-dimensional distributed feedback. Adv. Funct. Mater. 14, 91-97 (2004)

18. Vannahme, C., Klinkhammer, S., Lemmer, U. \& Mappes, T. Plastic lab-on-a-chip for fluorescence excitation with integrated organic semiconductor lasers. Opt. Express 19, 8179-8186 (2011).

19. Clark, J. \& Lanzani, G. Organic photonics for communications. Nat. Photonics 4, 438-446 (2010).

20. Cranch, G. A., Flockhart, G. M. H. \& Kirkendall, C. K. Distributed feedback fiber laser strain sensors. IEEE Sens. J. 8, 1161-1172 (2008).

21. Kallinger, C. et al. A flexible conjugated polymer laser. Adv. Mater. 10, 920-923 (1998).

22. Herrnsdorf, J. et al. Flexible blue-emitting encapsulated organic semiconductor DFB laser. Opt. Express 18, 25536-25545 (2010).

23. Kim, J. et al. Tunable and flexible solvent-free liquid organic distributed feedback lasers. Appl. Phys. Lett. 106, 053302 (2015).

24. Wenger, B., Tétreault, N., Welland, M. E. \& Friend, R. H. Mechanically tunable conjugated polymer distributed feedback lasers. Appl. Phys. Lett. 97, 193303 (2010).

25. Canazza, G. et al. Lasing from all-polymer microcavities. Laser Phys. Lett. 11, 035804 (2014).

26. Karl, M. et al. Flexible and ultra-lightweight polymer membrane lasers. Nat. Commun. 9, 1525 (2018).

27. Takeuchi, H. \& Sakata, H. Single mode lasing in polymeric distributed-feedback structure formed on inorganic distributed Bragg reflector. Laser Phys. Lett. 5, 41-44 (2008).

28. Lova, P. et al. Polymer distributed Bragg reflectors for vapor sensing. ACS Photonics 2, 537-543 (2015).

29. Tsutsumi, N., Nagi, S., Kinashi, K. \& Sakai, W. Re-evaluation of all-plastic organic dye laser with DFB structure fabricated using photoresists. Sci. Rep. 6, 34741 (2016).

30. Karnutsch, C. et al. Low threshold blue conjugated polymer lasers with first- and second-order distributed feedback. Appl. Phys. Lett. 89, 201108 (2006).
31. Yu, Z. et al. Fluorene-based copolymers containing branched 2-methylbutyl-substituted fluorene-co-benzothiadiazole units for remarkable optical gain enhancement in green-yellow emission range. J. Phys. Chem. C 120, 11350-11358 (2016).

32. Castro Smirnov, J. R. et al. Flexible all-polymer waveguide for low threshold amplified spontaneous emission. Sci. Rep. 6, 34565 (2016).

33. Xu, Y. et al. Efficient optical gain from near-infrared polymer lasers based on poly [N-9'-heptadecanyl-2,7-carbazole-alt- 5,5-(4',7'-di-2-thienyl-2', $1^{\prime}, 3^{\prime}$-benzothiadiazole)]. Adv. Opt. Mater. 6, 1800263 (2018).

34. El-Diasty, F. \& Bakry, A. M. Spectroscopic and sub-bandgap optical properties of gamma-irradiated cellulose triacetate polymer. J. Phys. D: Appl. Phys. 42, 145413 (2009).

35. Kosaka, P. M., Kawano, Y. \& Petri, D. F. S. Dewetting and surface properties of ultrathin films of cellulose esters. J. Colloid Interface Sci. 316, 671-677 (2007).

36. Rueda, D. R. et al. Grazing-incidence small-angle X-ray scattering of soft and hard nanofabricated gratings. J. Appl. Cryst. 45, 1038-1045 (2012).

37. Soccio, M. et al. On the assessment by grazing-incidence small-angle X-ray scattering of replica quality in polymer gratings fabricated by nanoimprint lithography. J. Appl. Cryst. 47, 613-618 (2014).

38. Yan, M. \& Gibaud, A. On the intersection of grating truncation rods with the Ewald sphere studied by grazing-incidence small-angle X-ray scattering. J. Appl. Cryst. 40, 1050-1055 (2007).

39. Pflüger, M., Soltwisch, V., Probst, J., Scholzea, F. \& Krumreya, M. GISAXS on small periodic targets using large beams. IUCrJ 4, 431-438 (2017).

40. Rabe, T. et al. Threshold reduction in polymer lasers based on poly(9,9-dioctylfluorene) with statistical binaphthyl units. Adv. Funct. Mater. 15, 1188-1192 (2005).

41. Zhang, Q. et al. Host exciton confinement for enhanced förster-transfer blend gain media yielding highly efficient yellow-green lasers. Adv. Funct. Mater. 28, 1705824 (2018).

42. Xia, R., Heliotis, G., Stavrinou, P. N. \& Bradley, D. D. C. Polyfluorene distributed feedback lasers operating in the green-yellow spectral region. Appl. Phys. Lett. 87, 031104 (2005)

43. Xia, R., Stavrinou, P. N., Bradley, D. D. C. \& Kim, Y. Efficient optical gain media comprising binary blends of poly(3-hexylthiophene) and poly(9,9-dioctylfluoreneco-benzothiadiazole). J. Appl. Phys. 111, 123107 (2012).

44. Huang, W., Chen, L. \& Xuan, L. Efficient laser emission from organic semiconductor activated holographic polymer dispersed liquid crystal transmission gratings. RSC Adv. 4, 38606-38613 (2014).

45. Chun, G., Meng, L., Xun, J., Yafang, T. \& Cunningham, B. T. Large-area organic distributed feedback laser fabricated by nanoreplica molding and horizontal dipping. Opt. Express 18, 12980-12991 (2010).

46. Lu, M., Choi, S. S., Irfan, U. \& Cunningham, B. T. Plastic distributed feedback laser biosensor. Appl. Phys. Lett. 93, 111113 (2008).

47. Xia, R., Heliotis, G., Hou, Y. \& Bradley, D. D. C. Fluorene-based conjugated polymer optical gain media. Org. Electron. 4, 165-167 (2003).

48. Stevens, M. A., Silva, C., Russell, D. M. \& Friend, R. H. Exciton dissociation mechanisms in the polymeric semiconductors poly(9,9-dioctylfluorene) and poly (9,9-dioctylfluorene-co-benzothiadiazole). Phys. Rev. B 63, 165213 (2001).

49. $\mathrm{Wu}, \mathrm{L}$. et al. Ground state host-guest interactions upon effective dispersion of regioregular poly (3-hexylthiophene) in poly (9, 9-dioctylfluorene-alt-benzothiadiazole). Macromolecules 48, 8765-8772 (2015).

50. Yi, J. et al. Optimized waveguide geometry for low amplified spontaneous emission operation on ito-coated glass. IEEE J. Sel. Top. Quantum Electron. 22, 21-25 (2016).

51. Campoy-Quiles, M. et al. Ellipsometric characterization of the optical constants of polyfluorene gain media. Adv. Funct. Mater. 15, 925-933 (2005).

52. Borsboom, M. et al. The Dutch-Belgian beamline at the ESRF. J. Synchrotron Radiat. 5, 518-520 (1998).

53. Müller-Buschbaum, P. Applications of synchrotron light to scattering and diffraction in materials and life sciences. Lect. Notes Phys. 776, 61-89 (2009).

Open Access This article is licensed under a Creative Commons Attribution 4.0 International License, which permits use, sharing, adaptation, distribution and reproduction in any medium or format, as long as you give appropriate credit to the original author(s) and the source, provide a link to the Creative Commons license, and indicate if changes were made. The images or other third party material in this article are included in the article's Creative Commons license, unless indicated otherwise in a credit line to the material. If material is not included in the article's Creative Commons license and your intended use is not permitted by statutory regulation or exceeds the permitted use, you will need to obtain permission directly from the copyright holder. To view a copy of this license, visit http://creativecommons. org/licenses/by/4.0/.

(c) The Author(s) 2019 